\title{
BMJ Open Pharmaceutical treatments to prevent recurrence of endometriosis following surgery: a model-based economic evaluation
}

\author{
Sabina Sanghera, ${ }^{1,2}$ Pelham Barton, ${ }^{1}$ Siladitya Bhattacharya, ${ }^{3}$ Andrew W Horne, ${ }^{4}$ \\ Tracy Elizabeth Roberts, ${ }^{1}$ on behalf of the PRE-EMPT research group
}

To cite: Sanghera S, Barton P, Bhattacharya S, et al. Pharmaceutical treatments to prevent recurrence of endometriosis following surgery: a modelbased economic evaluation. BMJ Open 2016;6:e010580. doi:10.1136/bmjopen-2015010580

- Prepublication history for this paper is available online. To view these files please visit the journal online (http://dx.doi.org/10.1136/ bmjopen-2015-010580)

Received 17 November 2015 Revised 10 March 2016 Accepted 17 March 2016

CrossMark

\footnotetext{
${ }^{1}$ Health Economics Unit, University of Birmingham, Birmingham, UK

${ }^{2}$ School of Social and Community Medicine, University of Bristol, Bristol, UK

${ }^{3}$ School of Medicine and Dentistry, University of Aberdeen, Aberdeen, UK ${ }^{4} \mathrm{MRC}$ Centre for Reproductive Health, University of Edinburgh, Edinburgh, UK

Correspondence to Professor Tracy Elizabeth Roberts; T.E.Roberts@bham. ac.uk
}

\begin{abstract}
Objective: Conduct an economic evaluation based on best currently available evidence comparing alternative treatments levonorgestrel-releasing intrauterine system, depot-medroxyprogesterone acetate, combined oral contraceptive pill (COCP) and 'no treatment' to prevent recurrence of endometriosis after conservative surgery in primary care, and to inform the design of a planned trial-based economic evaluation.
\end{abstract}

Methods: We developed a state transition (Markov) model with a 36-month follow-up. The model structure was informed by a pragmatic review and clinical experts. The economic evaluation adopted a UK National Health Service perspective and was based on an outcome of incremental cost per quality-adjusted life year (QALY). As available data were limited, intentionally wide distributions were assigned around model inputs, and the average costs and outcome of the probabilistic sensitivity analyses were reported.

Results: On average, all strategies were more expensive and generated fewer QALYs compared to no treatment. However, uncertainty attributing to the transition probabilities affected the results. Inputs relating to effectiveness, changes in treatment and the time at which the change is made were the main causes of uncertainty, illustrating areas where robust and specific data collection is required.

Conclusions: There is currently no evidence to support any treatment being recommended to prevent the recurrence of endometriosis following conservative surgery. The study highlights the importance of developing decision models at the outset of a trial to identify data requirements to conduct a robust posttrial analysis.

\section{INTRODUCTION}

Endometriosis is a chronic condition where tissue resembling that of the lining of the uterus (endometrium) grows in the pelvis and other areas of the body in response to ovarian hormones. ${ }^{1}$ The condition affects $2-10 \%$ of women of reproductive age, and it

\section{Strengths and limitations of this study}

- To our knowledge, this is the first study to conduct an economic evaluation of hormonal treatment for endometriosis following conservative surgery.

- The model-based analysis is preliminary but an integral component of a wider study comprising a randomised controlled trial.

- The limitations relate to the limited data available to determine which treatment should currently be recommended for treatment.

- Structural assumptions required for the model were not tested in a sensitivity analysis, as this would not have improved the precision of our findings.

- It was problematic to synthesise all available evidence for use in the model, so wide distributions were appropriately assigned to test the extent to which uncertainty in the parameters alters the results, and whether a trial is required.

is suggested that the economic impact of the condition is similar to other chronic diseases, such as diabetes mellitus and asthma. ${ }^{2}$ The predominant complaint in women with endometriosis is pelvic pain. ${ }^{1}$ While a reduction in pain is important to women, it is also important to assess the impact of treatment on general well-being and quality of life. ${ }^{1-4}$ Conservative surgical interventions that preserve fertility, such as laparoscopic removal or destruction of endometriotic tissue, are typically used to treat the condition. However, following surgery, there is an estimated $40-45 \%$ risk of symptom recurrence, increasing the likelihood of repeat surgery or more radical options such as hysterectomy. ${ }^{5}$ The underlying mechanisms explaining recurrence are uncertain. Recurrence may occur due to the regrowth of residual endometriotic lesions that were not completely removed in the surgery, growth of 
microscopic endometriosis undetected at surgery, or the development of de-novo lesions, or a combination of these. To eliminate or delay recurrence is presently an unmet medical need in the management of endometriosis.

In some small trials, hormonal therapy following conservative surgery has prevented or delayed recurrence of endometriosis. ${ }^{6}{ }^{7}$ The levonorgestrel-releasing intrauterine device (LNG-IUS) can be inserted during surgery, or subsequent to surgery by a general practitioner (GP), and is effective for 5 years. Other options include depotmedroxyprogesterone acetate (DMPA), an intramuscular injection that is administered every 3 months, and the combined oral contraceptive pill (COCP). In the absence of definitive clinical trials demonstrating evidence of effectiveness of one over the other, there is wide variation in their use which is dictated by personal preference and side effects. ${ }^{7}$

This study represents the preliminary component of an NIHR-funded study which includes a randomised controlled trial (RCT) (PRE-EMPT) (http://www. controlled-trials.com/ISRCTN97865475). Prior to this study, the applicants carried out a survey of the British Gynaecological Endoscopy Society members. The survey, which was reported in the funding application and the trial protocol, provided key information which was used to design the main RCT. It indicated that almost a quarter of clinicians used no postoperative treatment, and that the most commonly prescribed treatments are LNG-IUS, COCP and DMPA. It suggested that oral progestogens and GnRH analogues are not commonly prescribed. Thus, it was the three treatments of LNG-IUS, COCP, DMPA and 'no treatment' that were deemed to be the strategies most in need of evaluation. Furthermore, limiting the trial to just four main comparisons allowed a deliverable trial to be designed. The planned RCT will collect data to explore the most effective and cost-effective treatment for preventing endometriosis following surgery, but is not due to report for 4 years. To ensure that treatment decisions are based on robust evidence prior to trial data collection, it is important to explore key aspects of uncertainty associated with input parameters that feed into the trial evaluation. Therefore, a pretrial economic analysis is carried out to support immediate decisions, explore decision uncertainty and to inform areas of particular focus for data collection in the full RCT.

The aim of the preliminary component of the study was to (1) conduct an economic evaluation based on existing evidence for preventing endometriosis; (2) inform the design and analysis for the planned full trialbased economic evaluation. ${ }^{8}$

\section{METHODS}

We carried out a model-based economic evaluation which compared each hormonal treatment strategy, LNG-IUS, DMPA, COCP and no treatment in order to establish the most cost-effective approach for reducing symptom recurrence following conservative surgery for endometriosis based on the existing available evidence prior to the planned RCT. The evaluation took the form of a cost-utility analysis, based on an outcome of cost per quality-adjusted life year (QALY). A UK National Health Service perspective in a primary care setting was used. The analysis provides an assessment of the difference in costs and outcomes in terms of QALYs between the potential interventions over a 36-month time horizon. The time horizon reflects that of the relevant treatments that are required post-surgery and the need for treatment will be clear within that time period, it is also the time period on which the planned full RCT will be based.

\section{Model}

We developed a state transition (Markov) model in Microsoft Excel. The model structure was based on the NIHR-funded (PRE-EMPT study) RCT design, which was informed by a review of existing evidence and clinical input (http://www.controlled-trials.com/ ISRCTN97865475). The model structure was refined by iterative discussion with expert clinical colleagues. The initial structure was discussed with all clinicians associated with the planned RCT, disagreement was resolved by compromise, as practice varies considerably, and the final structure represents the most pragmatic structure which achieved consensus. Based on the results of our survey which informed the design of the planned RCT, we compared the same four alternative treatment strategies following conservative surgery: (1) no treatment, (2) LNG-IUS, (3) DMPA and (4) COCP. Where the first active treatment is deemed not effective, the patient is assumed to receive an additional round of hormonal treatment prior to having further surgery. Thus, as these treatments are not mutually exclusive, the pathway for each strategy is defined by the intervention that is tried first. Figure 1 shows the clinical pathway for the alternative treatment strategies. A monthly time cycle was used, as this was deemed to appropriately reflect changes in outcomes and resource use. We compared a cohort of 1000 women for each treatment strategy. The starting age was 32 years, based on the average age of a woman who has undergone conservative surgery for endometriosis. ${ }^{9}$ All-cause mortality was not considered due to the relatively short time horizon of the pretrial model. A half-cycle correction was not employed because transitions are deemed to occur at the end of the cycle as we are using a discrete model not a continuous model, therefore the use of the half-cycle correction would be incorrect. The following assumptions were made and developed based on clinical expertise from the PRE-EMPT trial research group. In developing the assumption, the group was careful to consider variation in practice which was likely to exist to ensure the assumptions were justified given potential variation. All assumptions were agreed prior to conducting the analysis. 


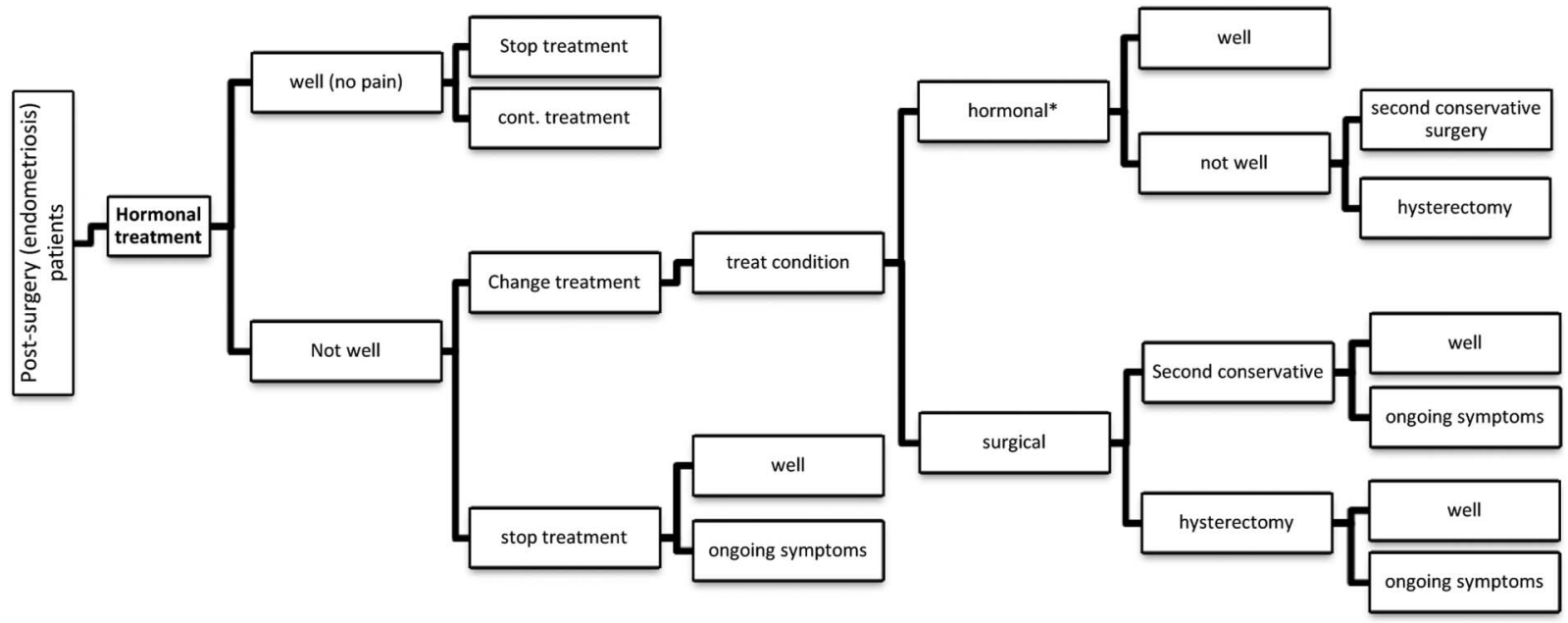

Figure 1 Clinical pathways for 'no treatment' and hormonal treatment $\left({ }^{*} F o r\right.$ 'hormonal' the treatment could be GnRHa or Mirena or COCP (33.3\% chance of each) - see assumptions. The pathway for hormonal treatment with GnRHa will be the same as the pathway in the hormonal treatment model). COCP, combined oral contraceptive pill; GnRHa, gonadotropin-releasing hormone analogue; LNG-IUS, levonorgestrel-releasing intrauterine device.

\section{Model assumptions}

- If a woman is symptomatic, and LNG-IUS is the initial treatment, the device will remain in situ for at least 12 months before it can be removed. We use the maximum amount of time suggested by clinicians for each treatment, to ensure the analysis was not favouring any particular treatment. It is assumed that LNG-IUS is inserted after surgical treatment for endometriosis

- If a woman is asymptomatic with LNG-IUS, the device is not removed for the remainder of the analysis

- If a woman is asymptomatic while receiving treatment with either COCP or DMPA, the treatment will not be stopped for at least 6 months. Following these 6 months, the woman can stop treatment, and it is assumed that the woman will remain asymptomatic without further treatment

- If symptomatic once off treatment, the woman will remain in this symptomatic state for 6 months before recommencing treatment or spontaneously becoming asymptomatic while off treatment. This enables sufficient time for previous hormonal effects to subside and time to determine if symptoms adjust or are resolved

- The second hormonal treatment will comprise one of the two remaining treatment strategies or GnRHa, which includes hormone replacement therapy, to enable the treatment to be used longer term than 6 months

- If symptomatic on the second hormonal treatment, the woman cannot have surgery until after 6 months have passed

- If asymptomatic on the second hormonal treatment, the woman will not stop treatment and will remain asymptomatic for at least 6 months

- A woman will undergo two sets of hormonal treatment before she can have either conservative surgery or hysterectomy 
- The impact of any of the treatments on long-term fertility has not been considered in the model

A pragmatic literature search was carried out to identify evidence on the effectiveness of the four treatment strategies to inform parameter inputs and their distributions for the transition probabilities of the decision model.

Thus, the evidence on current medical therapies described in the PRE-EMPT trial funding application and protocol was reviewed to identify data on effectiveness required for the transition probability parameters in the model. To identify literature for the utility values for the health states in the model, we searched the Web of Science database using the following key words 'endometriosis' and 'economic' and 'EQ-5D' or 'quality of life' or 'SF-6D'. A few small studies were identified that assessed the effectiveness of one of the treatment strategies, COCP, DMPA or LNG-IUS. ${ }^{10-14}$ Treatment effectiveness was typically measured in relation to pain and was captured using either the visual analogue scale (which provides a measure of quality of life), endometriosisspecific scale or the percentage improvement in a specific category. However, the available data were all based on small studies which used different measures for effectiveness, and so, synthesis into a meaningful analysis proved problematic. Additionally, data on disease recurrence after conservative surgery were extremely disparate. As a consequence of limited data availability in the published literature, transition probabilities were assigned by asking two of the trial clinicians to rank the likelihood of each subsequent event occurring from 1 to 5 or 1 to 3 , and distributions were assigned around these rankings to assess, as far as feasible, any uncertainty associated with the given values. These data are presented in table 1 . The intention was to assign distributions around the estimates which were deliberately wide to identify the extent to which changes in these values gave a different cost-effectiveness decision (outlined later) as is commonly the case for pretrial analyses where the purpose is to determine whether additional information is required to conduct a more robust analysis. ${ }^{15}$

\section{Utilities}

A search of the relevant literature did not identify any evidence on the effectiveness of the treatments in terms

Table 1 Probability parameters used in the analysis

\begin{tabular}{lll}
\hline Probability parameters & Distribution & Parameters \\
\hline LNG-IUS & & $(3,2)$ \\
LNG-IUS to asymptomatic on LNG-IUS & $\beta$ & $(3,2)$ \\
LNG-IUS to symptomatic on LNG-IUS & Fixed & 1 \\
Remain asymptomatic on LNG-IUS & Fixed & 1 \\
Remain asymptomatic off treatment & Fixed & 1 \\
Remain symptomatic on LNG-IUS (<12 months) & Dirichlet & 8 \\
Remain symptomatic on LNG-IUS (>12 months) & & 20 \\
Symptomatic with LNG-IUS to symptomatic off treatment & & 16 \\
Symptomatic with LNG-IUS to change treatment (hormonal) & & 4 \\
Symptomatic with LNG-IUS to hysterectomy & Fixed & 2 \\
Symptomatic with LNG-IUS to conservative surgery & Dirichlet & 1 \\
Remain symptomatic off treatment (<6 months) & & 6 \\
Remain symptomatic off treatment (>6 months) & $\beta$ & 2 \\
Symptomatic off treatment to asymptomatic off treatment & $\beta$ & 10 \\
Symptomatic off treatment to change treatment (hormonal) & 1 & $(3,2)$ \\
Change treatment (hormonal) to asymptomatic on 2nd treatment & $\beta$ & $(3,2)$ \\
Change treatment (hormonal) to symptomatic on 2nd treatment & $\beta$ & Fixed \\
Remain asymptomatic on 2nd treatment (<6 months) & $(3,2)$ \\
Remain asymptomatic on 2nd treatment (>6 months) & Fixed \\
Asymptomatic on 2nd treatment to asymptomatic off treatment & Dirichlet & $(3,2)$ \\
Remain symptomatic on 2nd treatment $(<6$ months) & & 1 \\
Remain symptomatic on 2nd treatment (>6 months) & & 6 \\
Symptomatic on 2nd treatment to 2nd conservative surgery & $\beta$ & 10 \\
Symptomatic on 2nd treatment to hysterectomy & $\beta$ & 2 \\
2nd conservative surgery to symptomatic post-conservative surgery & Fixed \\
2nd conservative surgery to asymptomatic post-conservative surgery & Fixed & $\beta$ \\
Remain symptomatic post-conservative surgery & $\beta$ & $(3,2)$ \\
Remain asymptomatic post-conservative surgery & Fixed & $(3,2)$ \\
Hysterectomy to symptomatic post-hysterectomy & Fixed & 1 \\
Hysterectomy to asymptomatic post-hysterectomy & & 1 \\
Remain symptomatic post-hysterectomy & & $(3,2)$ \\
Remain asymptomatic post-hysterectomy & $(3,2)$ \\
\hline
\end{tabular}

The same principle applies to the transitions for other treatments.

LNG-IUS, levonorgestrel-releasing intrauterine device. 
of utilities (which is a measure of preference for alternative health states) as measured by generic health-related quality of life instruments (ie, EQ-5D/ SF-6D) on which to base distributions. As a result of the dearth of evidence, clinicians were asked to rank the health states on a $0-10$ scale for the utilities associated with the treatments for endometriosis. The $0-10$ scale is analogous to the $0-1$ utility scale, and is thought to be easier to comprehend when providing estimates, so we used $0-10$ as a more pragmatic method for generating utilities. Similar to the transition probabilities and for the same reasons, an intentionally broad distribution was then assigned around these assumed point estimates with values in a range of 0.05 above and below. Where the clinicians disagreed with a given utility value, the range around the point estimate was broadened to account for this. Table 2 shows the estimated point estimates, the range used and the associated distribution to assess uncertainty around the given estimate.

\section{Cost and resource use}

The costs for all treatment strategies include healthcare staff costs and the cost of the intervention (table 3). The estimates for resource use were taken from a recent parallel primary study exploring the treatment for a related condition. ${ }^{16}$ Thus, LNG-IUS is estimated to take $20 \mathrm{~min}$ to fit, requires disposable items and both a GP and nurse to be present. Follow-up consultations for treatment with the GP are assumed to last $10 \mathrm{~min}$. Staff costs were calculated using nationally recognised reference costs. ${ }^{17}$ The costs of the treatments were estimated from the British National Formulary. ${ }^{18}$ Cost data on hysterectomy and second conservative surgery were taken from the literature and inflated to 2013 prices. ${ }^{19}$ As recommended by the National Institute for Health and Care Excellence, a discount rate of $3.5 \%$ was applied to both costs and utilities as the model time horizon is beyond 1 year. ${ }^{20}$ All costs are reported in 2013 prices in UK (£), using the UK hospital and community health services index. ${ }^{17}$ As the second hormonal treatment can be one of three treatments, $1 / 3$ of the average unit costs of three treatments are applied.

\section{Analysis}

In the analyses, we compared all four treatment strategies. Dominance is said to occur where a treatment is both less costly and more effective (ie, produces more QALYs) than another treatment. For each model input parameter (ie, effectiveness value, quality of life or utility value), a wide distribution is assigned to assess the extent of uncertainty associated with model inputs, and a value for each parameter is randomly drawn from the assigned distribution. This process, known as a probabilistic sensitivity analysis (PSA), simultaneously changes all relevant parameters in the model and is repeated 1000 times. To report an overall cost-effectiveness decision, the uncertainty around the input parameters was summarised as average costs and QALYs.

The 1000 values representing the ranges of incremental cost and QALYs for one treatment compared to

Table 2 Utility values used in analysis

\begin{tabular}{|c|c|c|c|c|}
\hline Health state & Estimate* $^{\star}$ & Range & Distribution† & Source \\
\hline \multicolumn{5}{|l|}{ All pathways } \\
\hline Baseline & 0.4 & $0.35-0.45$ & $\beta(2,2)$ & Symptomatic off treatment \\
\hline Asymptomatic off treatment & 1 & - & - & Ranking exercise \\
\hline Symptomatic off treatment & 0.4 & $0.35-0.45$ & $\beta(2,2)$ & Ranking exercise \\
\hline Conservative surgery & - & - & - & post-surgery \\
\hline Symptomatic post-conservative surgery & 0.35 & $0.25-0.45$ & $\beta(2,2)$ & Ranking exercise \\
\hline Asymptomatic post-conservative surgery & 0.8 & $0.75-0.85$ & $\beta(2,2)$ & Ranking exercise \\
\hline Hysterectomy & - & - & - & post-surgery \\
\hline Symptomatic post-hysterectomy & 0.25 & $0.15-0.35$ & $\beta(2,2)$ & Ranking exercise \\
\hline Asymptomatic post-hysterectomy & 0.7 & $0.65-0.75$ & $\beta(2,2)$ & Ranking exercise \\
\hline \multicolumn{5}{|l|}{ DMPA/COCP } \\
\hline Asymptomatic initial treatment & 0.7 & $0.65-0.75$ & $\beta(2,2)$ & Ranking exercise \\
\hline Symptomatic initial treatment & 0.35 & $0.25-0.45$ & $\beta(2,2)$ & Ranking exercise \\
\hline Change treatment (second treatment) & - & - & - & Symptomatic second treatment \\
\hline Asymptomatic second treatment & 0.7 & $0.65-0.75$ & $\beta(2,2)$ & Ranking exercise \\
\hline Symptomatic second treatment & 0.25 & $0.15-0.35$ & $\beta(2,2)$ & Ranking exercise \\
\hline \multicolumn{5}{|l|}{ LNG-IUS/no treatment } \\
\hline Asymptomatic on LNG-IUS & 0.8 & $0.75-0.85$ & $\beta(2,2)$ & Ranking exercise \\
\hline Symptomatic on LNG-IUS & 0.35 & $0.25-0.45$ & $\beta(2,2)$ & Ranking exercise \\
\hline Change treatment & - & - & - & Symptomatic second treatment \\
\hline Asymptomatic second treatment & 0.7 & $0.65-0.75$ & $\beta(2,2)$ & Ranking exercise \\
\hline Symptomatic second treatment & 0.3 & $0.25-0.35$ & $\beta(2,2)$ & Ranking exercise \\
\hline
\end{tabular}

*The estimate reflects the values provided by clinicians and is used only to set the range of values for the analysis.

†These $\beta$ distributions are applied within the ranges shown in the previous column, not across the full range of possible values from 0 to 1. Thus, the sampled value for any asymptomatic state will always be higher than the sampled value for the corresponding symptomatic state. COCP, combined oral contraceptive pill; DMPA, depot-medroxyprogesterone acetate; LNG-IUS, levonorgestrel-releasing intrauterine device. 
Table 3 Cost and resource use data used in analysis

\begin{tabular}{|c|c|c|}
\hline & Unit cost $(£)$ & Source \\
\hline \multicolumn{3}{|l|}{ LNG-IUS } \\
\hline Consultation (GP $10 \mathrm{~min}$ ) & 26.67 & Curtis $^{17} /$ Sanghera et $a l^{16}$ \\
\hline \multicolumn{3}{|l|}{ Insertion } \\
\hline GP (20 min) & 53.33 & Curtis $^{17} /$ Sanghera et $a l^{16}$ \\
\hline Practice nurse (20 $\mathrm{min})$ & 17.00 & Curtis $^{17} /$ Sanghera et al ${ }^{16}$ \\
\hline Device cost & 88.00 & BNF 66 \\
\hline Sterile pack (insertion) & 21.63 & NICE (inflated to 2011) \\
\hline \multicolumn{3}{|l|}{ Discontinuation } \\
\hline GP (10 min) & 26.67 & Curtis $^{17} /$ Sanghera et $a l^{16}$ \\
\hline Practice nurse (10 min) & 8.50 & Curtis $^{17} /$ Sanghera et $a l^{16}$ \\
\hline Sterile pack (removal) & 3.77 & NICE (inflated to 2013) \\
\hline \multicolumn{3}{|l|}{ Follow-up } \\
\hline 6 weeks review: (GP $10 \mathrm{~min}$ ) & 26.67 & Curtis $^{17} /$ Sanghera et $a l^{16}$ \\
\hline 3 months: (GP $10 \mathrm{~min}$ ) & 26.67 & Curtis $^{17} /$ Sanghera et $a l^{16}$ \\
\hline Combined oral contraceptive (microgynon) & 2.82 & BNF 66 \\
\hline Consultation: (GP $10 \mathrm{~min}$ ) & 26.67 & Curtis $^{17} /$ Sanghera et al $l^{16}$ \\
\hline 3 or 6 months review (GP $10 \mathrm{~min}$ ) & 26.67 & Curtis $^{17} /$ Sanghera et $a l^{16}$ \\
\hline Methoxyprogesterone acetate injections (Depo-provera) & 6.01 & BNF 66 \\
\hline Consultation: (GP $10 \mathrm{~min}$ ) & 26.67 & Curtis $^{17} /$ Sanghera et $a l^{16}$ \\
\hline 3 or 6 months review (GP $10 \mathrm{~min}$ ) & 26.67 & Curtis $^{17} /$ Sanghera et $a 1^{16}$ \\
\hline Discontinuation (GP $10 \mathrm{~min}$ ) & 26.67 & Curtis $^{17} /$ Sanghera et $a l^{16}$ \\
\hline Second conservative surgery & 1392.63 & Roberts et $a^{19}$ (inflated to 2013) \\
\hline Hysterectomy & 2432.04 & Roberts et al ${ }^{19}$ (inflated to 2013) \\
\hline
\end{tabular}

another are presented on the cost-effectiveness plane scatter plot to illustrate the distribution of values. These were used to construct a cost-effectiveness acceptability curve (CEAC) which shows the probability of each treatment being more cost-effective than the other, across a range of monetary values that decision-makers may be willing to pay for an additional QALY. We carried out a number of bilateral comparisons of treatment strategies, and therefore, generated several incremental costeffectiveness planes and CEACs.

The degree of uncertainty attributable to each parameter, that is, transition probability or utilities, was assessed by first holding the transition probabilities constant and allowing for variation around the utilities alone, and then second, by holding utilities constant and allowing for variation around the transition probabilities alone. This analysis was carried out by fixing parameters (ie, transition probabilities) to the last values that were obtained from a random draw of the distribution and allowing the parameters for the utilities to be randomly sampled from the assigned distribution and vice versa. Cost-effectiveness planes produced for each set of parameters varied, but it is inappropriate to use all these planes to produce CEACs, as they will not show the overall uncertainty. CEACs were only produced to illustrate the overall uncertainty.

\section{RESULTS}

The average costs and QALYs over the 1000 PSA iterations show that LNG-IUS is the most expensive intervention (£650.94) followed by DMPA (£622.56), then COCP (£599.93). No treatment is the least expensive intervention (£371.34), and also generated the greatest number of QALYs (2.27) followed by DMPA and COCP which both generated 1.92 QALYs, and LNG-IUS produced the least QALYs at 1.88.

The results show that no treatment dominates all other treatment strategies as it is both less costly and based on current evidence generates more QALYs. It can be seen from the scatter plots and the CEACs (figure 2, parts 1-3) that when compared with one another, there is little difference between the probability of each of the treatment strategies being cost-effective. There is clear uncertainty about which of the active treatment strategies are optimal. When the strategies are compared with no treatment, no treatment has a greater probability of being cost-effective at least $80 \%$ for all threshold willingness-to-pay values. The cost-effectiveness plane which illustrates the incremental costs and QALYs between the pairs of treatments shows that for every bilateral comparison of treatment strategies a negative slope is generated which crosses the origin indicating that there is considerable uncertainty around the findings. Table 4 presents a summary of the average results obtained from the PSA.

When utilities are held constant and variation is applied to the transition probabilities incremental costs, and QALYs again generate a negative slope on the costeffectiveness plane (figure 3, parts 1 and 2) which crosses the origin in every case, indicating a strong negative correlation between costs and QALYs, that is, the 
Figure 2 Illustrative results for average 1000 PSA iterations on the cost-effectiveness plane and the cost-effectiveness acceptability curve. COCP, combined oral contraceptive pill; DMPA, depotmedroxyprogesterone acetate; ICER, incremental costeffectiveness ratio; LNG-IUS, levonorgestrel-releasing intrauterine device; QALY, quality-adjusted life year.
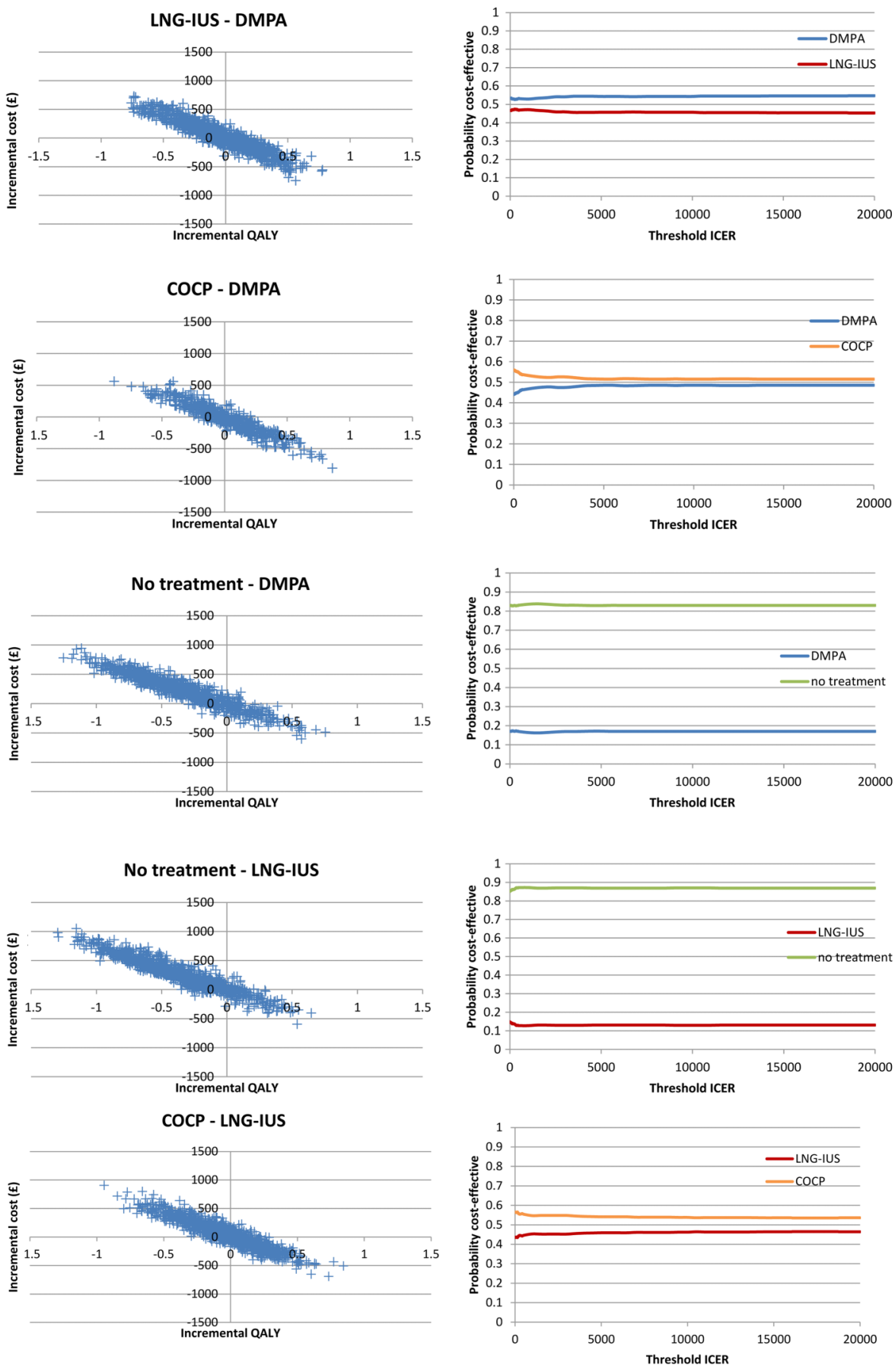

COCP - No treatment

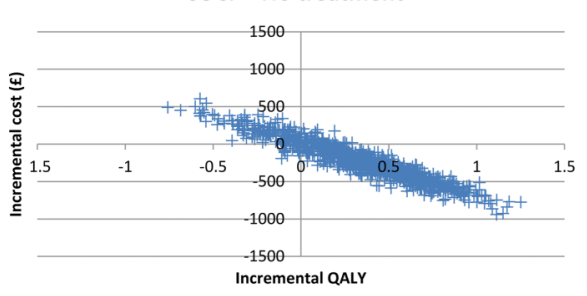

treatment could either be more costly and less effective, or more effective and less costly. Since costs are held constant, the most likely cause for this slope across the northwest and southeast quadrants of the costeffectiveness plane is the uncertainty around the transition probabilities. Therefore, inputs relating to effectiveness, changes in treatment and the time at which the change is made were the main causes of uncertainty, illustrating areas where robust and specific data collection is required. 
Table 4 Illustrative results averaged over 1000 PSA iterations

\begin{tabular}{llll}
\hline & Mean cost $(£)$ & Mean QALY & \\
\hline DMPA & 622.56 & 1.92 & Dominated \\
LNG-IUS & 650.94 & 1.88 & Dominated \\
COCP & 599.93 & 1.92 & Dominated \\
No treatment & 371.34 & 2.27 & \\
\hline
\end{tabular}

COCP, combined oral contraceptive pill; DMPA, depot-medroxyprogesterone acetate; LNG-IUS, levonorgestrel-releasing intrauterine device; PSA, probabilistic sensitivity analysis.

When transition probabilities are held constant and variation is applied around utilities (figure 4, parts 1 and 2), it can be seen that relative to the other treatments, the uncertainty is greatest around the quality of life values for LNG-IUS. In all the bilateral comparisons including LNG-IUS (LNG-IUS/DMPA, no treatment/ LNG-IUS and COCP/LNG-IUS) the scatter of incremental costs and QALYs cross the axis of the costeffectiveness plane to varying degrees, indicating that there is uncertainty around the most cost-effective treatment in the comparisons.

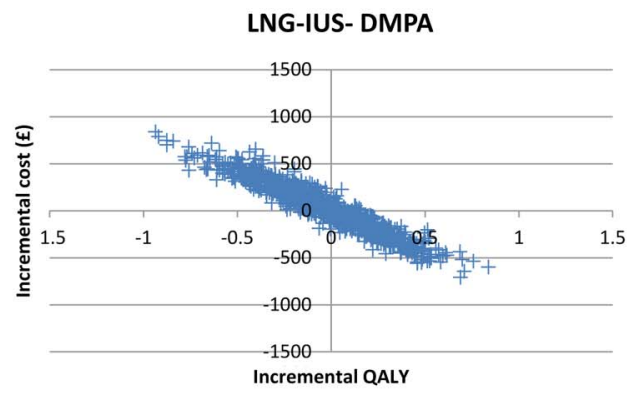

No treatment - DMPA

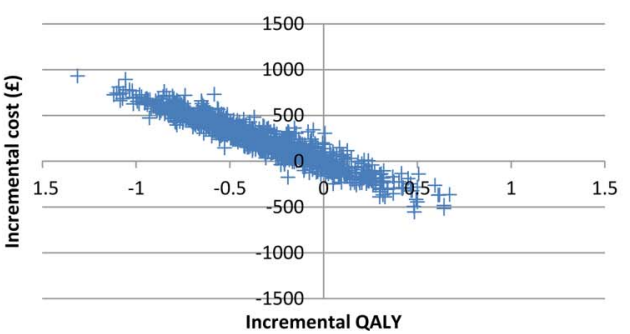

COCP - LNG-IUS

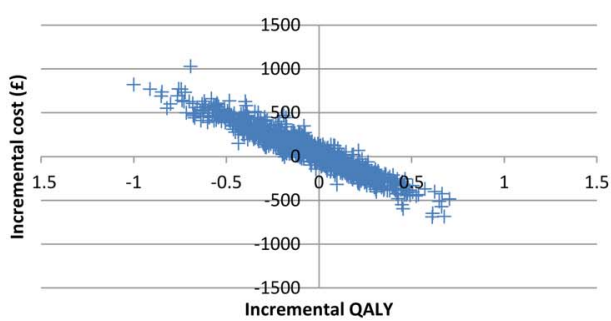

\section{DISCUSSION}

\section{Main findings}

The main findings from this study are that there is considerable uncertainty about the cost-effectiveness of the existing treatments to prevent endometriosis following conservative surgery. The model-based analysis, based on best available current data, suggests that no treatment is the appropriate course of action to prevent recurrence of endometriosis following conservative surgery when compared with other treatment strategies (DMPA, COCP and LNG-IUS). This result appears to be driven by the assumption that being on treatment will itself lead to a substantial reduction in quality of life. There is little difference between the probability of all existing treatment strategies (DMPA, COCP, LNG-IUS) being the most cost-effective, and none can yet be singled out as a potential clear contender. In terms of the direction of results, COCP had the greatest probability of being costeffective when compared with DMPA and LNG-IUS. DMPA had the highest probability of being cost effective when compared with LNG-IUS.

While no treatment should be recommended at the current time for this clinical condition, the transition probabilities, which relate to the effectiveness of the

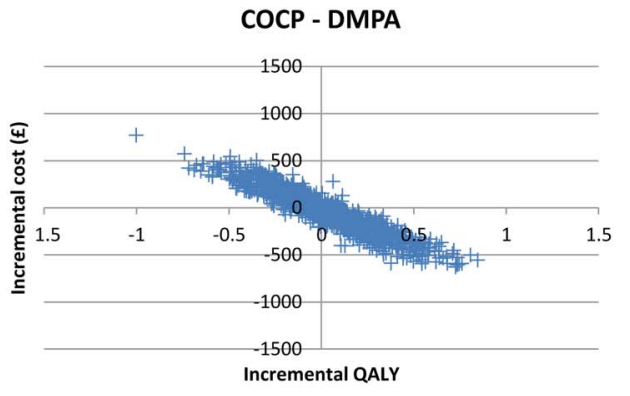

No treatment - LNG-IUS

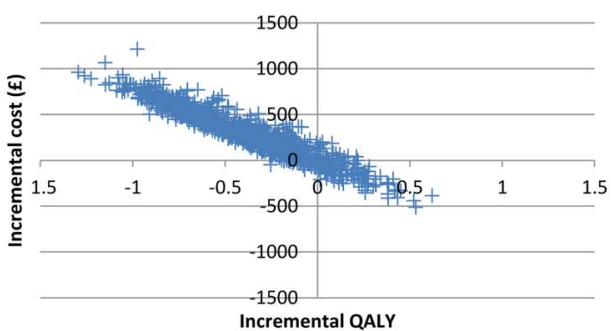

COCP - No treatment

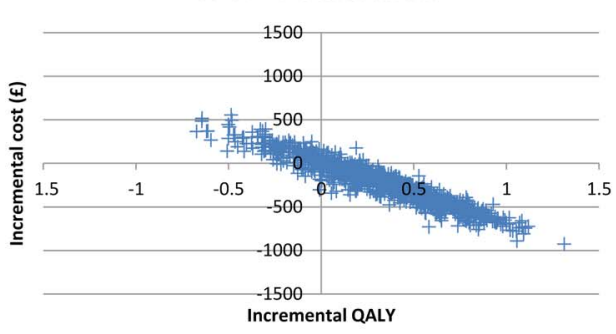

Figure 3 PSA results on the cost-effectiveness plane where only transition probabilities are probabilistic. COCP, combined oral contraceptive pill; DMPA, depot-medroxyprogesterone acetate; LNG-IUS, levonorgestrel-releasing intrauterine device; QALY, quality-adjusted life year. 
LNG-IUS- DMPA

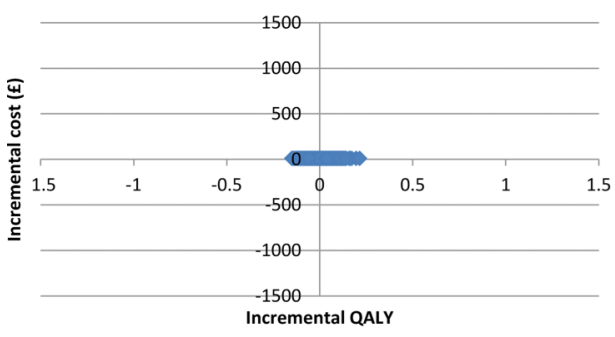

No treatment - DMPA

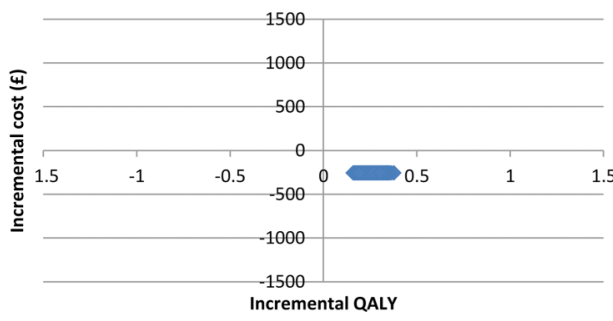

COCP - LNG-IUS

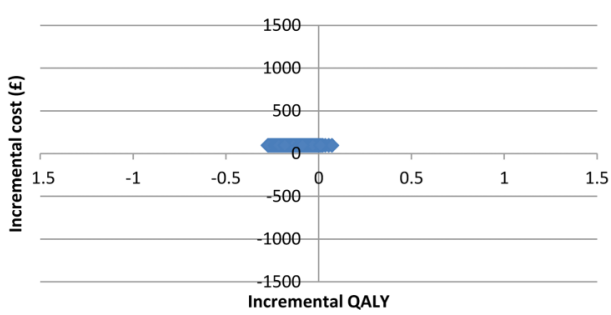

COCP - DMPA

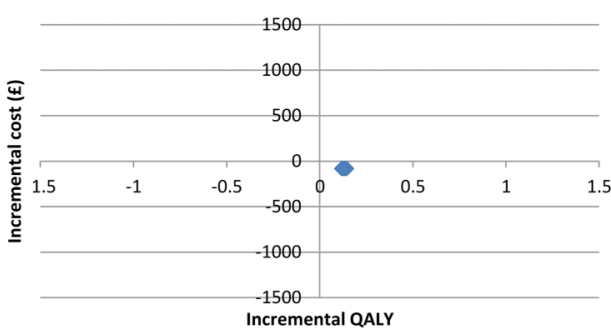

No treatment - LNG-IUS

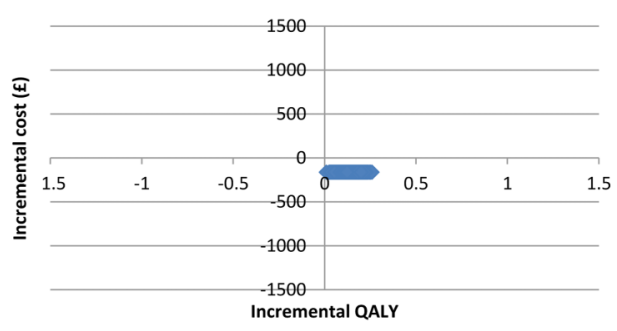

COCP - No treatment

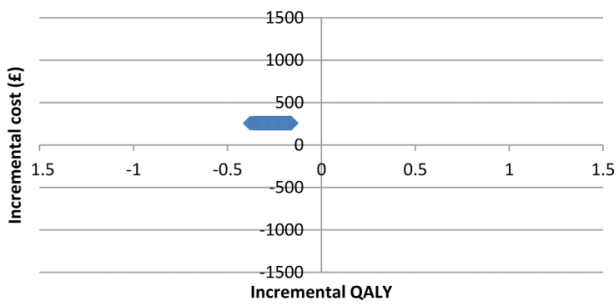

Figure 4 PSA results on the cost-effectiveness plane where only utilities are probabilistic. COCP, combined oral contraceptive pill; DMPA, depot-medroxyprogesterone acetate; LNG-IUS, levonorgestrel-releasing intrauterine device; QALY, quality-adjusted life year.

treatment, are subject to a great deal of uncertainty, which is reflected in the results. This wholly supports the need for further primary research to allow a more conclusive recommendation to be made. The full planned trial is necessary to resolve the uncertainty. The study highlights the importance of developing decision models at the outset of a trial to identify data requirements to conduct a robust post-trial analysis. Areas in which the trial should focus data collection are on the effectiveness of LNG-IUS for this condition, the quality of life impact of the treatments, and the type and timing of treatment changes.

\section{Strengths and limitations}

We identified no other economic evaluations that assess the cost-effectiveness of these treatment strategies for postsurgical treatment of endometriosis. To our knowledge, this is the first study to conduct an economic evaluation of hormonal treatment for endometriosis following conservative surgery. The model-based analysis reported here is preliminary but an integral component of a wider study comprising an RCT. The current analysis is the first to identify and highlight issues that can be addressed and data that need to be collected in the trial to enhance the robustness of the trial-based cost-effectiveness analysis, and will aid the development of the model structure for the subsequent analysis.

The limitations of this model were that there were no reliable data available to determine which treatment should currently be recommended for treatment. Also, structural assumptions required for the model, such as allowing women to have three courses of hormonal treatment prior to surgery rather than two, were not tested in a sensitivity analysis, as this analysis would not have improved the precision of our findings but will be appropriate for the economic evaluation alongside the trial. However, these are not limitations that impact on a purpose of the pretrial analysis to inform data collection. Further, the data for the transition probabilities found in the literature were based on small studies, and the results were based on different measures. Thus, it was problematic to synthesise this evidence for use in the model. Wide distributions were appropriately assigned to test the extent to which uncertainty in the parameters alters the results and whether a trial is required. Illustrative data were used: given that the impact of the treatment on this particular condition is subjective, wide distributions around values seemed most suitable. Owing to the pragmatic approach to searching the literature, it is possible that additional usable studies could be found 
from a comprehensive systematic review. While it is unlikely a systematic review would reveal enough additional information to have a much higher level of confidence on parameter values, it cannot be ruled out.

\section{CONCLUSION}

As there is clear uncertainty around the findings, it is important to ensure the most robust evidence on the effectiveness of these three treatment strategies for endometriosis is acquired. In the meantime, until the trial results or other new evidence emerges, no treatment is recommended for the clinical condition following conservative surgery. As data are lacking on the effectiveness of these treatments for endometriosis it would be beneficial to compare the three treatment strategies compared in this analysis with a no treatment strategy as four different arms of the trial. The findings also suggest that it is essential to generate reliable evidence on the effectiveness of LNG-IUS for this condition, as the uncertainty around the utility, or quality of life values, has shown to generate both positive and negative incremental QALYs. In all cases, it is important to determine how best to capture quality of life data, and to identify which measure appropriately captures patient preferences. In addition to the typical data that are captured as part of the trial, it will be important to focus data collection to clarify specifically the type of treatment change made, if any, and the time (in months) at which the treatment change occurred. Such data should include whether and when the patient stopped treatment. It is also likely that the trial time horizon may need to be longer than 3 years for the full benefit of treatments to be seen.

This study suggests that at the current time there is no evidence to support any treatment being offered for this condition. However, the study illustrates the benefits and importance of conducting a model-based economic evaluation based on available evidence prior to a trialbased economic evaluation being carried out. ${ }^{8}$ The model-based economic evaluation provides information on areas where specific and reliable data collection is required to improve the robustness of the economic evaluation.

Twitter Follow Tracy Roberts at @tracyrobertsbham and Sabina Sanghera at @SabinaSanghera

Acknowledgements The authors would like to thank the other clinicians involved in the PRE-EMPT study who assisted us with the assumptions; Hilary Critchley, Andrew Prentice, Kevin Cooper.

Contributors SB, AWH and TER contributed to the design of the whole project and obtained funding. TER designed the economic component of the project to include a pretrial analysis. SS under the supervision of PB and TER conducted the pretrial analysis with advice from SB and AWH. SS prepared the manuscript as lead writer and TER edited the manuscript. All authors have commented on and approved the submitted manuscript.

Funding This work was supported by NIHR HTA grant number 11/114/01.

Competing interests None declared.
Provenance and peer review Not commissioned; externally peer reviewed.

Data sharing statement No additional data are available.

Open Access This is an Open Access article distributed in accordance with the Creative Commons Attribution Non Commercial (CC BY-NC 4.0) license, which permits others to distribute, remix, adapt, build upon this work noncommercially, and license their derivative works on different terms, provided the original work is properly cited and the use is non-commercial. See: http:// creativecommons.org/licenses/by-nc/4.0/

\section{REFERENCES}

1. Kennedy S, Bergqvist A, Chapron C, et al. ESHRE guideline for the diagnosis and treatment of endometriosis. Hum Reprod 2005;20:2698-704.

2. Eskenazi B, Warner ML. Epidemiology of endometriosis. Obstet Gynecol Clin North Am 1997;24:235-58.

3. ESHRE-European Society of Human Reproduction and Embryology. Management of women with endometriosis. ESHRE Guideline development group. 2013. http://www.eshre.eu/ /media/ Files/ESHRE\%20guideline\%20on\%20endometriosis\%202013\% 20v1.pdf

4. De Graaff AA, D'Hooghe TM, Dunselman GAJ, et al. The significant effect of endometriosis on physical, mental and social wellbeing: results from an international cross-sectional survey. Hum Reprod 2013;28:2677-85.

5. Garry R. The effectiveness of laparoscopic excision of endometriosis. Curr Opin Obstet Gynecol 2004;16:299-303.

6. Abou-Setta AM, Al-Inany HG, Farquhar CM. Levonorgestrel-releasing intrauterine device (LNG-IUD) for symptomatic endometriosis following surgery. Cochrane Database Syst Rev 2006;(4):CD005072.

7. Daniels JP, Clark TJ, Bhattacharya S. BSGE Survey of current post-operative hormonal treatment for Endometriosis. 2012.

8. Sculpher MJ, Claxton K, Drummond M, et al. Whither trial-based economic evaluation for health care decision making? Health Econ 2006;15:677-87.

9. Nnoaham KE, Hummelshoj L, Webster P, et al. Impact of endometriosis on quality of life and work productivity: a multicenter study across ten countries. Fertil Steril 2011;96:366-73.

10. Crosignani PG, Luciano A, Ray A. Subcutaneous depot medroxyprogesterone acetate versus leuprolide acetate in the treatment of endometriosis-associated pain. Hum Reprod 2006;21:248-56.

11. Sesti F, Pietropolli A, Capozzolo T, et al. Hormonal suppression treatment or dietary therapy versus placebo in the control of painful symptoms after conservative surgery for endometriosis stage III-IV. A randomized comparative trial. Fertil Steril 2007;88:1541-7.

12. Bayoglu Tekin Y, Dilbaz B, Altinbas SK, et al. Postoperative medical treatment of chronic pelvic pain related to severe endometriosis: levonorgestrel-releasing intrauterine system versus gonadotropinreleasing hormone analogue. Fertil Steril 2011;95:492-6.

13. Guzick DS, Huang LS, Broadman BA, et al. Randomized trial of leuprolide versus continuous oral contraceptives in the treatment of endometriosis-associated pelvic pain. Fertil Steril 2011;95:1568-73.

14. Tanmahasamut $P$, Rattanachaiyanont $M$, Angsuwathana $S$, et al. Postoperative levonorgestrel-releasing intrauterine system for pelvic endometriosis-related pain: a randomized controlled trial. Obstet Gynecol 2012;119:519-26.

15. Briggs A, Claxton K, Sculpher M. Decision modelling in health economic evaluation. Oxford: Oxford University Press, 2006.

16. Sanghera S, Roberts TE, Barton P, et al. Levonorgestrel-releasing intrauterine system vs. usual medical treatment for menorrhagia: an economic evaluation alongside a randomised controlled trial. PLOS ONE 2014;9:e91891.

17. Curtis L. Unit costs of health and social care. Personal Social Service Research Unit, 2013.

18. Joint Formulary Committee. British National Formulary 66. BMJ Group \& Pharmaceutical Press, 2013.

19. Roberts TE, Tsourapas A, Middleton LJ, et al. Hysterectomy, endometrial ablation, and levonorgestrel releasing intrauterine system (Mirena) for treatment of heavy menstrual bleeding: cost effectiveness analysis. BMJ 2011;342:d2202.

20. National Institute of Health and Clinical Excellence. Guide to the methods of technology appraisal. London: National Institute of Health and Clinical Excellence, 2008. 